\title{
Error characteristics of a model-based integration approach for fixed-wing unmanned aerial vehicles
}

\author{
Hery A. Mwenegoha, ${ }^{1 *}$ (D) Terry Moore, ${ }^{1}$ (D) James Pinchin, ${ }^{1}$ and Mark Jabbal ${ }^{2}$ \\ ${ }^{1}$ Nottingham Geospatial Institute, University of Nottingham, UK. \\ ${ }^{2}$ Fluids and Thermal Engineering Research Group, University of Nottingham, UK. \\ *Corresponding author. E-mail: hery.mwenegoha1@ nottingham.ac.uk
}

Received: 23 September 2020; Accepted: 21 April 2021

Keywords: vehicle dynamic model, model-based navigation, VDM, INS

\begin{abstract}
The paper presents the error characteristics of a vehicle dynamic model (VDM)-based integration architecture for fixed-wing unmanned aerial vehicles. Global navigation satellite system (GNSS) and inertial measurement unit measurements are fused in an extended Kalman filter (EKF) which uses the VDM as the main process model. Control inputs from the autopilot system are used to drive the navigation solution. Using a predefined trajectory with segments of both high and low dynamics and a variable wind profile, Monte Carlo simulations reveal a degrading performance in varying periods of GNSS outage lasting $10 \mathrm{~s}, 20 \mathrm{~s}, 30 \mathrm{~s}, 60 \mathrm{~s}$ and $90 \mathrm{~s}$, respectively. These are followed by periods of re-acquisition where the navigation solution recovers. With a GNSS outage lasting less than $60 \mathrm{~s}$, the position error gradually grows to a maximum of $8.4 \mathrm{~m}$ while attitude errors in roll and pitch remain bounded, as opposed to an inertial navigation system (INS)/GNSS approach in which the navigation solution degrades rapidly. The model-based approach shows improved navigation performance even with parameter uncertainties over a conventional INS/GNSS integration approach.
\end{abstract}

\section{Introduction}

Over the last few years, low-cost, mass-market unmanned aerial vehicles (UAVs) have seen increasing usage in 'dull, dangerous and dirty' (D-D-D) fields. The most common navigation architecture in these platforms is based on an inertial navigation system (INS) integrated with a global navigation satellite system (GNSS) (Kim and Sukkarieh, 2003; George and Sukkarieh, 2005). With an INS/GNSS architecture, problems tend to arise during GNSS outages where the navigation solution drifts unboundedly (Hide, 2003; Quinchia et al., 2013; Mwenegoha et al., 2019). This has been reported to occur due to intentional or unintentional corruption, even against cryptographically secured signals (Papadimitratos and Jovanovic, 2008), rapid dynamics (Tawk et al., 2014), severe multipath (Robustelli and Pugliano, 2018), loss of line of sight and interference (Groves, 2008).

To improve the navigation solution during GNSS outages, El-Diasty and Pagiatakis (2009) explored advanced inertial measurement unit (IMU) error modelling schemes. The approach showed some improvements in navigation performance at the expense of additional software complexities. Further, the approach used a standard INS/GNSS integration architecture using an unscented Kalman filter that would still be disabled in case of IMU failure. Lau et al. (2013) used acceleration white noise biases to model accelerometer errors to improve navigation performance during short GNSS outages. The architecture relied on conventional INS/GNSS integration, which would disable the navigation solution in case of IMU failure. Jospin et al. (2019) proposed using a cooperative navigation scheme using two 
UAVs in areas with severe multipath such as dams or near bridges. The approach used high-power light-emitting diodes (LEDs) on one platform positioned using a camera mounted on a second platform. Even though significant improvements in position estimation were achieved, the approach has limited application to fixed-wing UAVs, and the performance can be degraded in poor visibility conditions.

More recently, model-aided and model-based integration architectures using the vehicle dynamic model (VDM) fused with IMU and GNSS measurements have gained research popularity (Bryson and Sukkarieh, 2004; Dadkhah et al., 2008; Mwenegoha et al., 2019). A model-aided integration scheme employs an INS as the main process model and uses the VDM as an aiding tool while a model-based scheme uses the VDM as the main process model and an INS as an aiding system. A detailed discussion about the two concepts can be found in Mwenegoha et al. (2019). Crocoll et al. (2014) implemented a unified INS and VDM scheme and achieved significant improvements in navigation performance over a conventional INS/GNSS integration scheme. The authors showed that the approach was similar to the multi-process model scheme proposed by Koifman and Bar-Itzhack (1999) but computationally efficient by eliminating duplicate states. Experimental tests (Mueller et al., 2016) using a quadrotor showed significant improvement in position estimation during GNSS outages; however, the navigation solution could still be disabled in case of IMU failure. Sendobry (2014) proposed a model-based scheme and avoided duplicate states. The approach is similar to the one proposed by Khaghani and Skaloud (2016) and Mwenegoha et al. (2019) even though both studies were applied to a fixed-wing UAV while Sendobry considered a quadrotor. An experiment using a ground vehicle and a simplified VDM showed improved navigation performance near buildings; however, the impact of dynamics on navigation performance during GNSS outages was not investigated.

A model-based scheme can still output a navigation solution with useful attitude information even with an IMU failure when aided by a GNSS receiver, making it more favourable than a model-aided scheme (Khaghani and Skaloud, 2018). However, the characterisation of navigation solution errors during different lengths of GNSS outages has not been investigated. Further, the error characteristics with varying roll rates during turns has also not been explored. Therefore, this paper presents findings of the error characteristics of a model-based approach in different GNSS outage lengths and varying roll rates, especially during turns. Comparisons with a conventional INS/GNSS approach are also made.

\section{Model-based architecture}

Figure 1 shows the model-based architecture investigated compared with a conventional INS/GNSS integration scheme which is more common in low-cost UAVs. The model-based architecture uses the dynamic model of a fixed-wing UAV as the main process model coupled with IMU and GNSS measurements using an extended Kalman filter (EKF). The architecture uses rotation rate and specific force measurements from the IMU as well as position measurements from the GNSS receiver in the fusion filter to estimate corrections to the navigation states. The control inputs from the autopilot system are used to propagate the navigation states. Mass and moment of inertia are assumed to be known and constant during the flight setup. The VDM requires a set of parameters $\left(X_{P}\right)$ used to derive the moments and forces acting on the aircraft, as can be seen in Figure 2. Pre-calibration of these parameters before a flight is possible but this can be time-consuming and usually requires expensive equipment.

The VDM structure enables the inflight calibration of these parameters reducing the effort in obtaining accurate parameters. Figure 3 shows the coordinate frames considered in the investigation alongside the control surface deflections. Table 1 shows the formulation details for the airspeed, angle of attack $(\alpha)$ and side-slip angle $(\beta)$, respectively.

\section{Simulation setup and equations of motion}

A Monte Carlo simulation study with 50 simulations is used to study the error characteristics of the architecture in Matlab. More details on the Monte Carlo simulation will be given in the next section. 


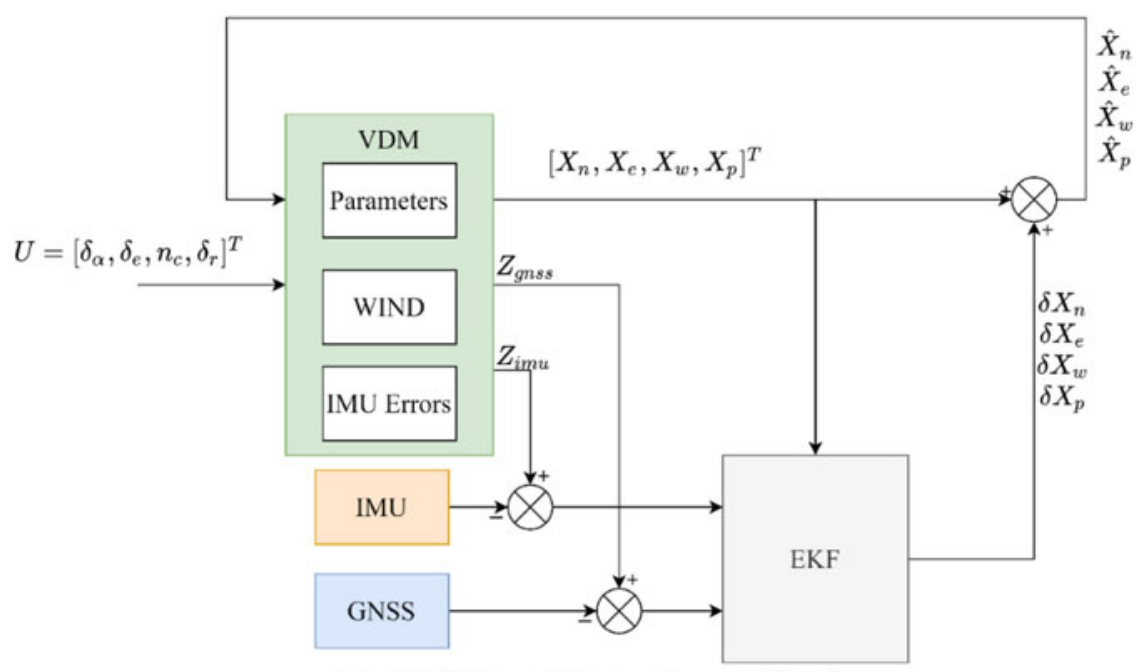

(a) VDM-based integration architecture

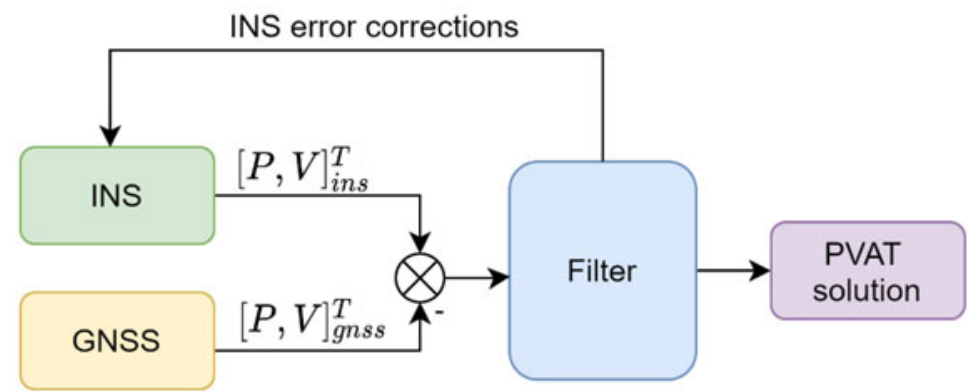

(b) INS/GNSS integration architecture

Figure 1. (a) VDM-based integration architecture, where $\delta_{\alpha}, \delta_{e}, n_{c}, \delta_{r}$ represent the aileron, elevator, propeller speed command and rudder deflection, respectively; $Z_{g n s s}, Z_{\text {imu }}$ represent the GNSS and IMU measurement model; $X_{n}, X_{e}, X_{w}, X_{p}$ represent the navigation states, IMU error states, wind error states and VDM parameter states, respectively. (b) INS/GNSS integration architecture, where PVAT represent the position, velocity, attitude and time solution; $[P, V]_{\text {ins }}^{T}$ represent the position and velocity solution from the INS and $[P, V]_{\text {gnss }}^{T}$ is output from the GNSS receiver.

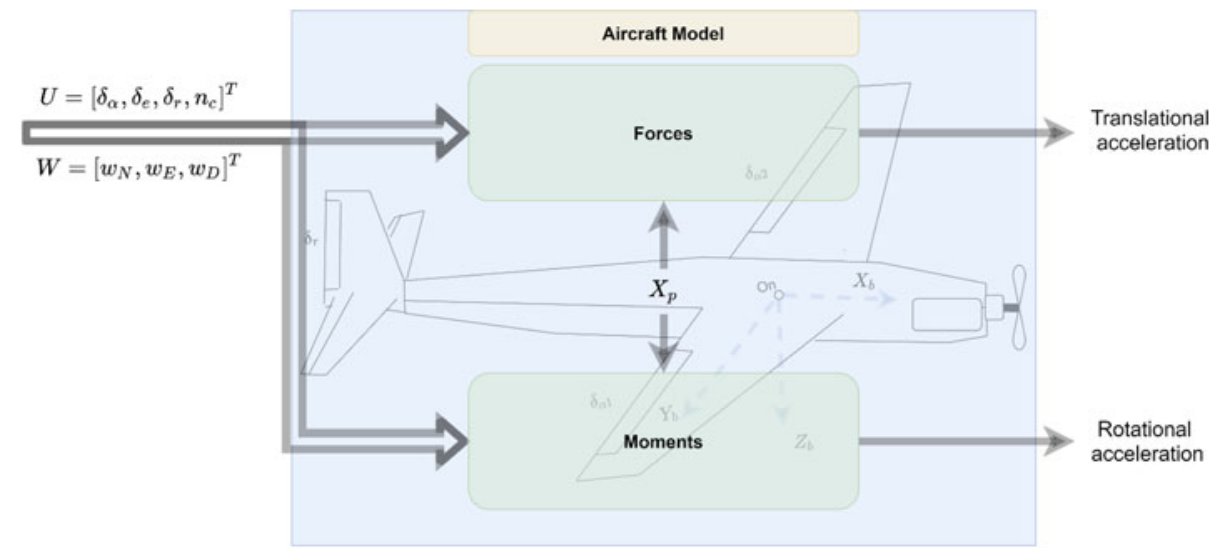

Figure 2. Diagram of VDM. It requires control inputs and wind velocity vector as inputs, which translate to translational and rotational accelerations that are integrated to propagate the navigation states. 


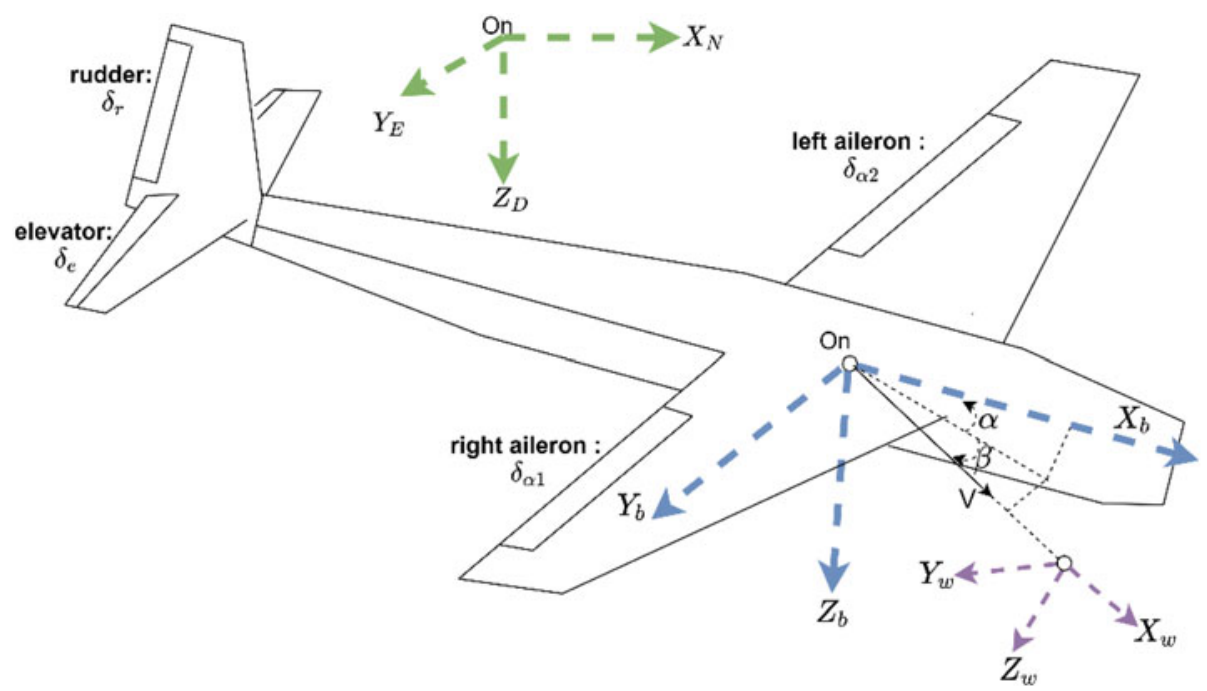

Figure 3. Coordinate frames used in the formulation of the equations of motion. $\left[\begin{array}{lll}X & Y & Z\end{array}\right]_{[b, w}$ represent the body frame $(b)$ and wind frame $(w)$ axes, respectively.

Table 1. Airspeed, angle of attack and side-slip angle.

\begin{tabular}{lcc}
\hline Airspeed $(V)$ & Angle of attack $(\alpha)$ & Side-slip angle $(\beta)$ \\
\hline$v^{b}=V^{b}+R_{n}^{b} W^{n}$ & $\alpha=\arctan \left(\frac{V_{z}^{b}}{V_{x}^{b}}\right)$ & $\beta=\arcsin \left(\frac{V_{y}^{b}}{V}\right)$ \\
$V^{b}=\left[\begin{array}{lll}b & V_{y}^{b} & V_{z}^{b}\end{array}\right]^{T}$ & & \\
$V=\left\|V^{b}\right\|$ & & \\
\hline
\end{tabular}

Note: $R_{n}^{b}$ : local level (NED) to body rotation matrix; $W^{n}$ : wind velocity vector in the NED frame.

The VDM navigation states considered in the simulation are:

$$
X_{n}=\left[r_{e b}^{n}, v_{e b}^{n}, \phi_{n b}, \omega_{i b}^{b}, n\right]^{T}
$$

where $r_{e b}^{n}=\left[\begin{array}{ll}\mu \lambda & h_{b}\end{array}\right]^{T}$ is the curvilinear position vector representing latitude, longitude and geodetic height, respectively. The velocity vector in the north-east-down (NED) frame is given by:

$$
v_{e b}^{n}=\left[v_{N}, v_{E}, v_{D}\right]^{T} .
$$

The Euler angles, roll, pitch and yaw are given by:

$$
\phi_{n b}=[\phi, \theta, \psi]^{T}
$$

and the rotation rate vector $\omega_{i b}^{b}=\left[\omega_{x}, \omega_{y}, \omega_{z}\right]^{T}$ represents the rotation around the roll, pitch and yaw axis. An arbitrary vector $V_{\alpha \beta}^{\gamma}$ represents a vector in the $\beta$ frame (object frame) with respect to the $\alpha$ frame (reference frame) resolved into the $\gamma$ frame (resolving frame). $n$ is the propeller speed implemented as 
a first-order process. The rigid body equations of motion are given by:

$$
\begin{aligned}
& \dot{r}_{e b}^{n}=\left[\frac{v_{e b, N}^{n}}{R_{M}+h}, \frac{v_{e b, E}^{n}}{\left(R_{P}+h\right) \cos \mu},-v_{e b, D}^{n}\right]^{T} \\
& \dot{v}_{e b}^{n}=R_{b}^{n} f_{i b}^{b}+g^{n}-\left(2 \Omega_{i e}^{n}+\Omega_{e n}^{n}\right) v_{e b}^{n} \\
& \dot{\phi}=R_{\phi}\left(\omega_{i b}^{b}-R_{n}^{b}\left(\omega_{i e}^{n}+\omega_{e n}^{n}\right)\right) \\
& R_{\phi}=\left[\begin{array}{ccc}
1 & \tan \theta \sin \phi & \tan \theta \cos \phi \\
0 & \cos \phi & -\sin \phi \\
0 & \frac{\sin \phi}{\cos \theta} & \frac{\cos \phi}{\cos \theta}
\end{array}\right] \\
& \dot{\omega}_{i b}^{b}=\left(I^{b}\right)^{-1}\left(M_{b}-\Omega_{i b}^{b}\left(I^{b} \omega_{i b}^{b}\right)\right) \\
& \dot{n}=\frac{\left(-n+n_{c}\right)}{\tau_{n}}
\end{aligned}
$$

where $g^{n}$ is the gravity vector in the local NED frame, $I^{b}$ is the mass moment of inertia matrix, $n_{c}$ and $\tau_{n}$ represent the commanded propeller speed and time constant, respectively. The meridian $\left(R_{M}\right)$ and prime vertical $\left(R_{P}\right)$ radius of curvature are given by:

$$
R_{M}=\frac{R_{0}\left(1-e^{2}\right)}{\left(1-e^{2} \sin ^{2}(\mu)\right)^{3 / 2}} \text { and } R_{P}=\frac{R_{0}}{\left(1-e^{2} \sin ^{2}(\mu)\right)^{1 / 2}}
$$

where $R_{0}$ and $e$ represent the semimajor axis and eccentricity, respectively. The specific force vector in the body frame $\left(f_{i b}^{b}\right)$ is given by:

$$
\begin{aligned}
f_{i b}^{b} & =\frac{1}{m}\left(R_{w}^{b}\left[\begin{array}{c}
F_{X}^{w} \\
F_{Y}^{w} \\
F_{Z}^{w}
\end{array}\right]+\left[\begin{array}{c}
F_{T} \\
0 \\
0
\end{array}\right]\right) \\
R_{w}^{b} & =\left[\begin{array}{ccc}
\cos \alpha \cos \beta & -\cos \alpha \sin \beta & -\sin \alpha \\
\sin \beta & \cos \beta & 0 \\
\cos \beta \sin \alpha & -\sin \alpha \sin \beta & \cos \alpha
\end{array}\right]
\end{aligned}
$$

where $F_{X}^{w}, F_{Y}^{w}, F_{Z}^{w}$ represent drag force, lateral force and lift force, respectively. $F_{T}$ is the thrust force and $R_{w}^{b}$ is the transformation matrix from the wind frame to the body frame. The moment term $\left(M_{b}\right)$ in the body frame is given by:-

$$
M_{b}=\left[M_{X}, M_{Y}, M_{Z}\right]^{T}
$$

where $M_{X}, M_{Y}, M_{Z}$ represent the roll, pitch and yaw moments, respectively. Table 2 further represents the forces and moments acting on the aircraft using the VDM parameters. The actual values for the VDM parameters used in this work can be found in Ducard (2007).

The aerodynamic and propulsion model presented in Table 2 describing the forces and moments acting on the aircraft can be found in Ducard (2007) and Khaghani and Skaloud (2016).

\section{Fusion filter and implementation}

An EKF is used in the fusion of GNSS and IMU measurements with the VDM. Navigation states $X_{n}$ are propagated using the presented equations of motion. IMU errors $\left(X_{e}\right)$, wind velocity states $\left(X_{w}\right)$ and VDM parameters $\left(X_{p}\right)$ are propagated using a random walk process (Mwenegoha et al., 2019). These 
Table 2. Forces and moments acting on the aircraft.

\begin{tabular}{ll}
\hline Forces & \multicolumn{1}{c}{ Moments } \\
\hline $\begin{array}{ll}F_{X}^{w}=\bar{q} S C F_{X} \\
C F_{X}=C F_{X 1}+C F_{X_{\alpha}} \alpha+C F_{X_{\alpha 2}} \alpha^{2}\end{array}$ & $M_{X}^{b}=\bar{q} S b C M_{X}$ \\
$\quad+C F_{X_{\beta 2}} \beta^{2}$ & $C M_{X}=C M_{X_{\delta_{\alpha}}} \delta_{\alpha}+C M_{X_{\bar{\omega}_{x}}} \bar{\omega}_{x}+C M_{X_{\bar{\omega}_{z}}} \bar{\omega}_{z}+C M_{X_{\beta}} \beta$ \\
$F_{Y}^{w}=\bar{q} S C F_{Y}$ & $M_{Y}^{b}=\bar{q} S \bar{c} C M_{Y}$ \\
$C F_{Y}=C F_{Y 1} \beta$ & $C M_{Y}=C M_{Y_{e}} \delta_{e}+C M_{\bar{\omega}_{y}} \bar{\omega}_{y}+C M_{Y_{\alpha}} \alpha+C M_{Y 1}$ \\
$F_{Z}^{w}=\bar{q} S C F_{Z}$ & $M_{Z}^{b}=\bar{q} S b C M_{Z}$ \\
$C F_{Z}=C F_{Z 1}+C F_{Z_{\alpha}} \alpha$ & $C M_{Z}=C M_{Z_{\delta_{r}}} \delta_{r}+C M_{Z_{\bar{\omega}_{z}}} \bar{\omega}_{z}+C M_{Z_{\beta}} \beta$ \\
$F_{T}=\rho n^{2} D^{4} C F_{T}$ & \\
$C F_{T}=C F_{T 1}+C F_{T 2} J+C F_{T 3} J^{2}$ & \\
$J=\frac{V}{D \pi n}$ & $\bar{\omega}_{x}=\frac{\omega_{x} b}{2 V}, \bar{\omega}_{z}=\frac{\omega_{z} b}{2 V}, \bar{\omega}_{y}=\frac{\omega_{y} \bar{c}}{2 V}$ \\
\hline
\end{tabular}

Note: $D$ : propeller diameter; $b$ : wingspan; $\rho$ : air density; $\bar{c}$ : mean aerodynamic chord.

states are given by:

$$
\begin{aligned}
& X_{e}=\left[b_{a_{1}}, b_{a_{2}}, b_{a_{3}}, b_{g_{1}}, b_{g_{2}}, b_{g_{3}}\right]^{T} \\
& X_{w}=\left[w_{N}, w_{E}, w_{D}\right]^{T}
\end{aligned}
$$

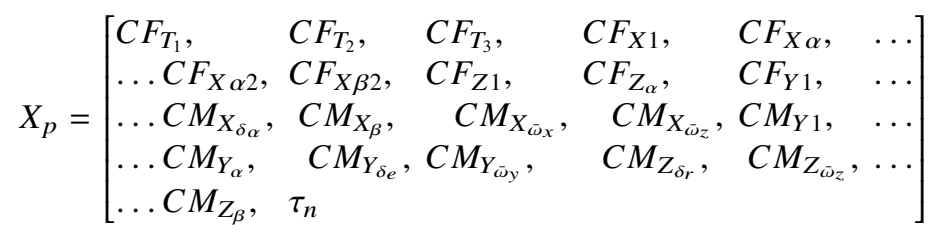

The general form of the random walk process model for the IMU error states, wind velocity states and VDM parameters used in the navigation system is given by:

$$
\dot{X}_{[e, w, p]}=G_{[e, w, p]} W_{[e, w, p]}
$$

where $G_{[e, w, p]}$ is the noise shaping matrix for the IMU error states, wind velocity states and VDM parameters, respectively. $W_{[e, w, p]}$ is the driving noise vector for the IMU error states, wind velocity states and VDM parameters, respectively.

In the simulator, the IMU errors and wind model follow a first-order Gauss-Markov process, and their parameters are unknown to the filter while the VDM parameters were fixed. The first-order GaussMarkov wind model used had a constant component with a magnitude of $3.8 \mathrm{~m} / \mathrm{s}$, a correlation time of $200 \mathrm{~s}$, and a process uncertainty of $0 \cdot 1 \mathrm{~m} / \mathrm{s}$. Generally, a first-order Gauss-Markov process $\left(X_{g m}\right)$ can be represented as:

$$
\dot{X}_{g m}=-\beta_{g m} X_{g m}+n_{g m}
$$

where $\beta_{g m}$ is the inverse of the correlation time and $n_{g m}$ is the driving noise.

The overall state vector is given by:

$$
x=\left[X_{n}, X_{e}, X_{w}, X_{p}\right]^{T}
$$

The measurement vector consists of IMU $\left(\tilde{f}_{i b}^{b}, \tilde{\omega}_{i b}^{b}\right)$ and GNSS $\left(\tilde{r}_{e b}^{n}\right)$ measurements.

$$
Z=\left[Z_{\mathrm{IMU}}, Z_{\mathrm{GNSS}}\right]^{T}
$$


Table 3. EKF propagation and update.

\begin{tabular}{ll}
\hline Propagation & \multicolumn{1}{c}{ Update } \\
\hline$\left[\delta \hat{X}_{k-1}^{+}, P_{k-1}^{+}\right]$ & $\delta \hat{X}_{k}^{+}=\delta \hat{X}_{k}^{-}+K\left(Z-h_{m}\left(X_{k}^{-}, U_{k}\right)\right)$ \\
$\delta \hat{X}_{k}^{-}=\Phi_{k-1} \delta \hat{X}_{k-1}^{+}$ & \\
$P_{k}^{-}=\Phi_{k-1} P_{k-1}^{+} \Phi_{k-1}^{T}+Q_{k-1}$ & $P_{k}^{+}=(I-K H) P_{k}^{-}(I-K H)^{T}+K R K^{T}$ \\
$\Phi_{k-1}=\sum_{m=0}^{\infty} \frac{F_{k-1}^{m} \tau_{s}^{m}}{m !}$ & $K=\frac{P_{k}^{-} H^{T}}{H P_{k}^{-} H^{T}+R}$ \\
$\Phi_{\mathrm{k}-1} \approx I+F_{k-1} \tau_{s}$ & $H=\partial Z / \partial X$ \\
\hline
\end{tabular}

where each measurement $(Z)$ is given by a measurement function $h$ :

$$
Z_{k}=h\left[x_{k}, u_{k}\right]+r_{k}
$$

where $x_{k}$ is the predicted state vector at the current time index $k, u_{k}$ is the known control input vector from the autopilot system and $r_{k}$ is the measurement noise where the expectation operator on the white sequence vector and its transpose is given by:

$$
E\left[r_{k} r_{k}^{T}\right]=R_{k}
$$

where $R_{k}$ is the measurement covariance. Therefore, the observation model for the IMU is given by:

$$
Z_{\mathrm{IMU}}=\left[\begin{array}{c}
f_{i b}^{b}+X_{e}\left(\left[\begin{array}{lll}
1 & 2 & 3
\end{array}\right]\right) \\
\omega_{i b}^{b}+X_{e}\left(\left[\begin{array}{lll}
4 & 5 & 6
\end{array}\right]\right)
\end{array}\right]+w_{i}
$$

where the IMU measurement covariance $\left(R_{i_{k}}=E\left[w_{i_{k}} w_{i_{k}}^{T}\right]\right)$ is obtained from the simulated error characteristics presented in Table 4. The observation model for the GNSS measurements is given by:

$$
Z_{\mathrm{GNSS}}=\left[\begin{array}{l}
\mu \\
\lambda \\
h
\end{array}\right]+w_{g}
$$

where the GNSS measurement covariance $\left(R_{g k}=E\left[w_{g k} w_{g k}^{T}\right]\right)$ is obtained from simulated GNSS receiver error with minor scaling. The filter utilises the linearised version of the process model $(F=$ $(\partial \dot{x} / \partial x))$ and observation model $(H=(\partial Z / \partial x))$ on the prediction and update of the state vector and the covariance matrix. The observation matrix $H$ is given by:

$$
\begin{aligned}
H & =\frac{\partial Z}{\partial x}=\frac{\partial\left[\begin{array}{lll}
Z_{\text {imu }} & Z_{\text {gnss }}
\end{array}\right]^{T}}{\partial\left[\begin{array}{llll}
X_{n} X_{e} & X_{w} & X_{p}
\end{array}\right]} \\
& =\left[\begin{array}{llll}
H_{\text {imu }, n} & H_{\text {imu }, e} & H_{\text {imu }, w} & H_{\text {imu }, p} \\
H_{\text {gnss }, n} & H_{\text {gnss }, e} & H_{\text {gnss }, w} & H_{\text {gnss }, p}
\end{array}\right]
\end{aligned}
$$

The EKF propagation and update steps are summarised in Table 3. Table 4 presents the stochastic properties of the sensors used in the simulation.

The trajectory used in the simulation is presented in Figure 4. The presented equations of motion, aerodynamic and propulsion models are implemented in Matlab/Simulink to generate the trajectory. The trajectory partly captures what would be experienced in a typical mapping or surveying mission. GNSS outages are induced during certain segments of the trajectory where the aircraft experiences rapid dynamics in roll, pitch, yaw or a combination of them, i.e., during turns. 
Table 4. Stochastic properties of the IMU and GNSS receiver.

\begin{tabular}{lll}
\hline Sensor & \multicolumn{1}{c}{ Type } & \multicolumn{1}{c}{ Value } \\
\hline Accelerometer & Random bias $(\sigma)$ & $10 \mathrm{mg}$ \\
& White noise (PSD) & $100 \mu \mathrm{g} / \sqrt{\mathrm{Hz}}$ \\
& GM-process & $0.05 \mathrm{mg}$ \\
& Correlation time $(\tau)$ & $200 \mathrm{~s}$ \\
Gyroscope & Sampling frequency & $100 \mathrm{~Hz}$ \\
& Random bias $(\sigma)$ & $1000 \circ / \mathrm{hr}$ \\
\hline & White noise (PSD) & $21.6 \% / \mathrm{hr} / \sqrt{\mathrm{Hz}}$ \\
& GM-process & $20 \% / \mathrm{hr}$ \\
GNSS receiver & Correlation time $(\tau)$ & $200 \mathrm{~s}$ \\
& Sampling frequency & $100 \mathrm{~Hz}$ \\
& White noise $(\sigma)$ & $5 \mathrm{~m}$ \\
\hline
\end{tabular}
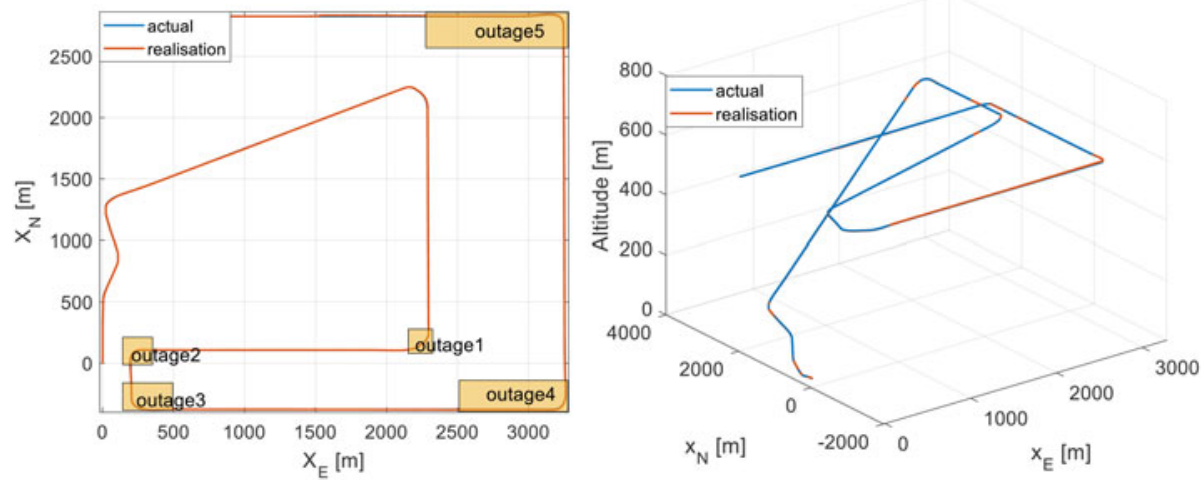

Figure 4. Trajectory used to study the error characteristics of a VDM/INS/GNSS architecture.

To evaluate the performance of the navigation system, a Monte Carlo simulation study has been performed with 50 runs. Errors are introduced to all the a priori information available such as the initial values for the navigation states, VDM parameters, statistics of the IMU and GNSS measurements. The trajectory and wind profile have been kept the same in each realisation. The error in observations, initialisation and VDM parameters is changed randomly in each run. VDM parameters are changed with a standard deviation of $10 \%$ of the initial values. The position errors for 50 Monte Carlo runs are presented in Figure 5.

A total of three simulations with the VDM architecture are performed with the given trajectory and three different autopilot settings. The autopilot limits the rotation rates to $15 \% \mathrm{~s}, 30 \% \mathrm{~s}$ and $60 \%$, respectively. Figure 6 shows the GNSS outage and re-acquisition segments as well as the roll rates and roll angles achieved during the three runs. The outage period is set to $10 \mathrm{~s}, 20 \mathrm{~s}, 30 \mathrm{~s}, 60 \mathrm{~s}$ and $90 \mathrm{~s}$, respectively during each simulation run, as seen in Figure 6.

\section{Error characteristics results}

The results from a Monte Carlo simulation study are presented in this section for the VDM approach and some comparison is made with an INS/GNSS approach. The initial $250 \mathrm{~s}$ of the flight are used for convergence and therefore removed from the discussion. 

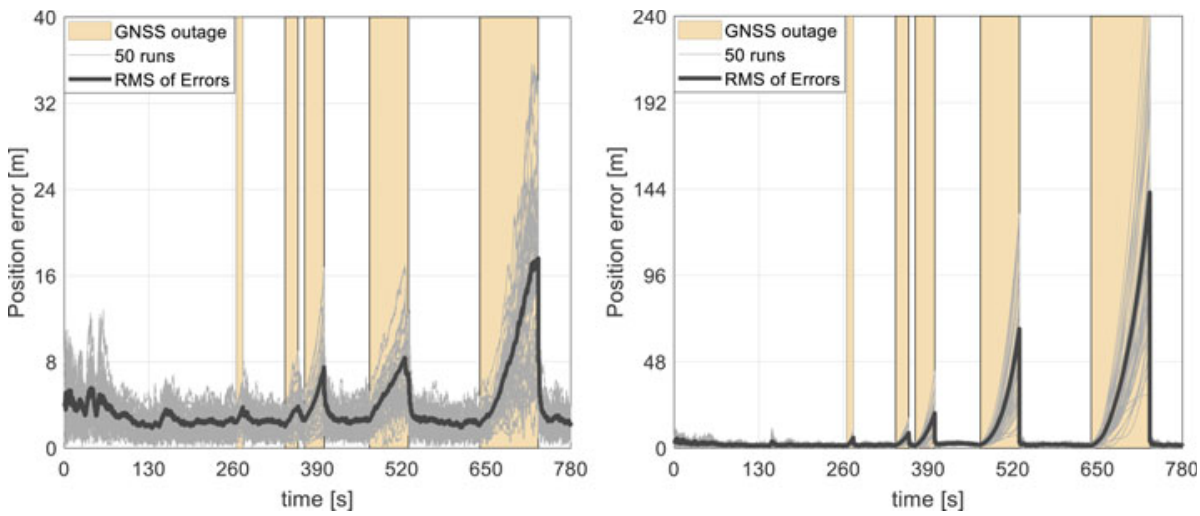

Figure 5. Position errors for 50 Monte Carlo runs for the VDM/INS/GNSS (left) scheme and the INS/GNSS (right) integration architecture.
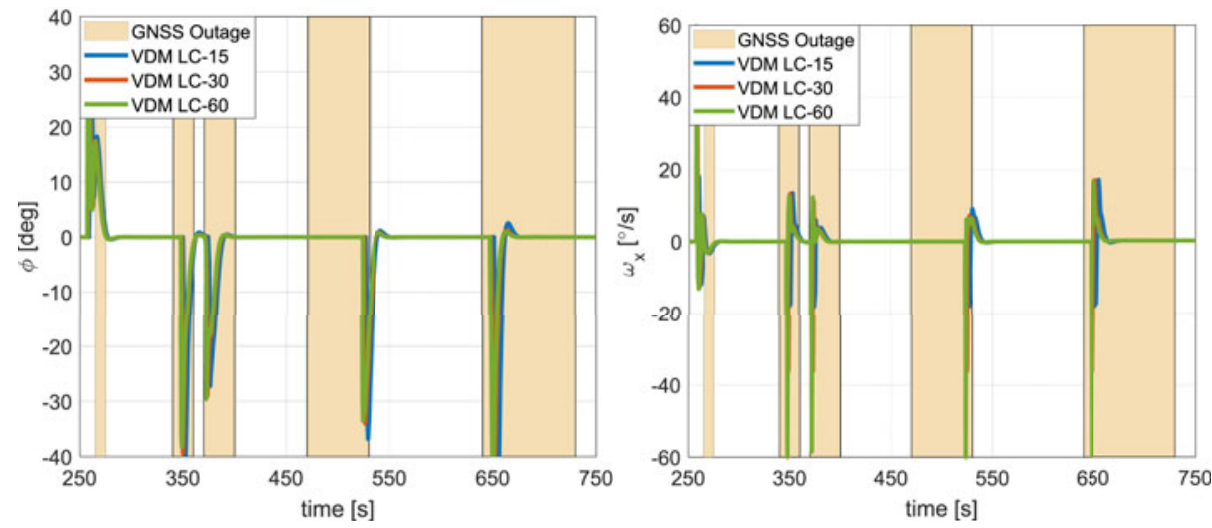

Figure 6. Dynamics in terms of roll angle (left) and roll rate (right) for the three simulation runs. VDM LC-15 represents the simulation run with $15 \%$ rate limit; VDM LC-30 represents the simulation run with $30 \%$ sate limit; VDM LC-60 represents the simulation run with $60 \%$ s rate limit.

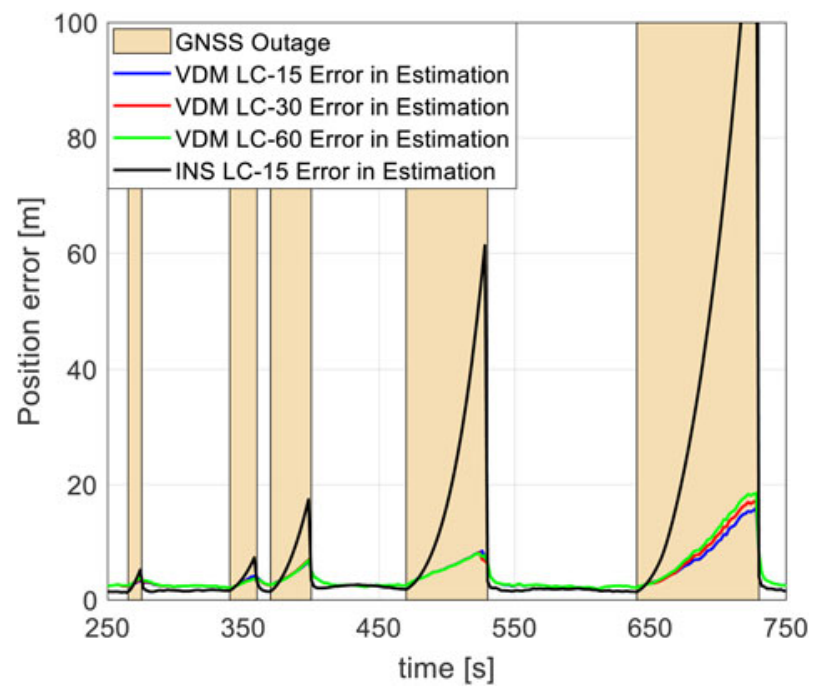

Figure 7. RMS of position error for VDM versus INS approach. 

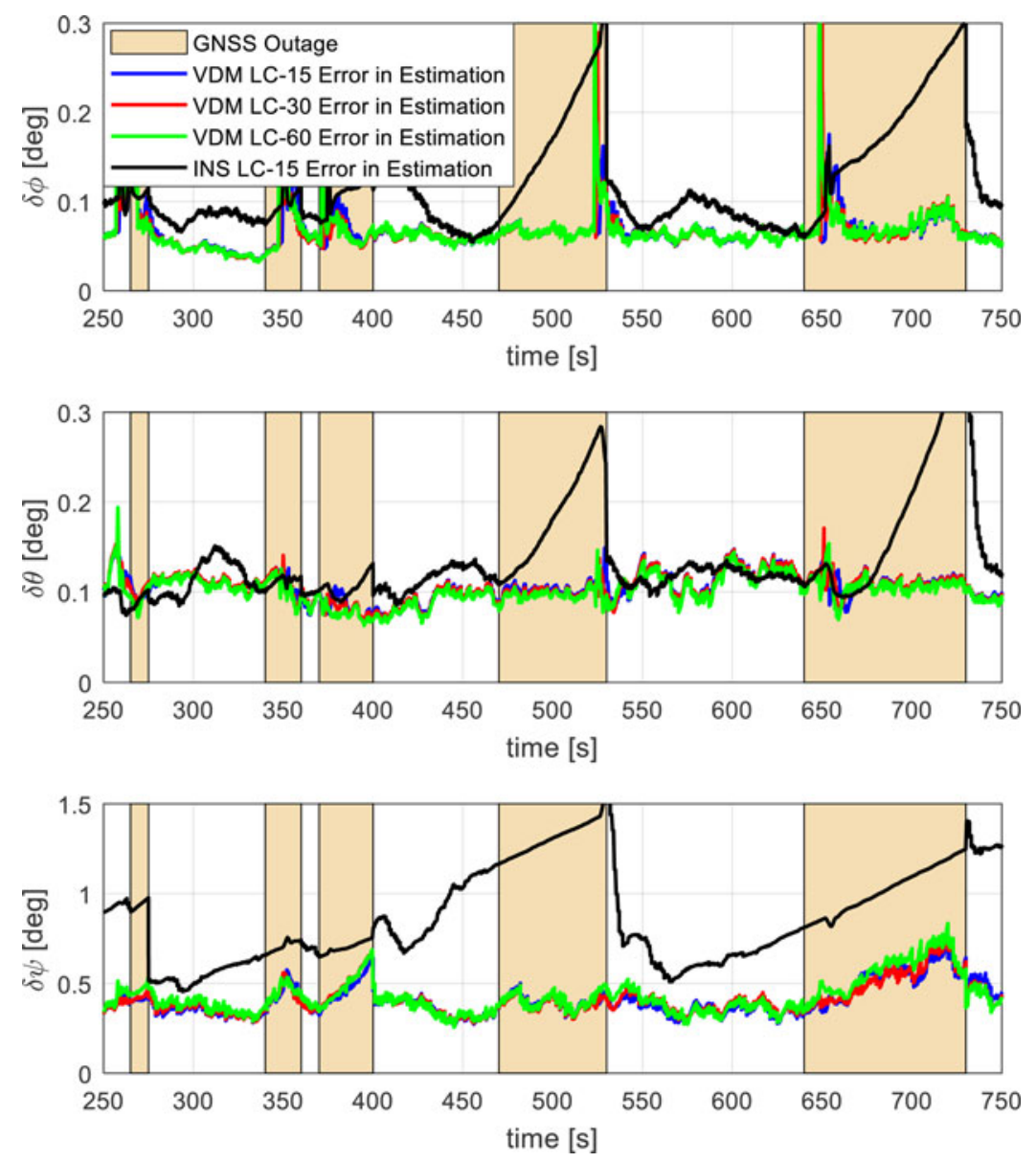

Figure 8. Attitude errors for VDM versus INS approach.

The position error is presented in Figure 7. For GNSS outages lasting up to $60 \mathrm{~s}$, the results showed that the position error for the VDM approach with different rotation rate limits was similar. The position errors for the different rate cases reached only $8.494 \mathrm{~m}$ after $60 \mathrm{~s}$ of GNSS outage, well within the $2 \sigma$ of the GNSS receiver modelled as opposed to $61 \mathrm{~m}$ for an INS/GNSS case. For GNSS outages lasting up to $90 \mathrm{~s}$, the level of dynamics mostly around the roll axis seemed to influence position error, as shown in Figure 7 in the last outage phase. With a rate limit of $60 \%$, the maximum position error was $16 \%$ greater than the position error observed with a rate limit of $15 \%$ and only $8 \%$ greater with a rate limit of $30 \%$ s.

With a GNSS outage lasting $90 \mathrm{~s}$, the position error of the INS/GNSS approach with a rate limit of $15 \%$ s was an order of magnitude higher than the VDM approach limited to $60 \%$ s. The additional information from using the VDM reduced the rapid drift in the navigation solution, leading to superior performance by the VDM approach even with rapid roll dynamics.

The root mean square (RMS) of the attitude errors is presented in Figure 8. For the VDM approach with different rotation rate limits, the overall roll and pitch angle errors during all periods of GNSS outage did not significantly change, excluding sections with rapid roll dynamics owing to the use of the VDM. However, yaw angle error seemed to increase gradually during an outage period, especially with some channel dynamics during the outage. Yaw angle error was found to increase, reaching a maximum value of 0.53 degrees, 0.55 degrees, 0.68 degrees and 0.8344 degrees during GNSS outages lasting $10 \mathrm{~s}, 20 \mathrm{~s}, 30 \mathrm{~s}$ and $90 \mathrm{~s}$, respectively. The GNSS outage period lasting $60 \mathrm{~s}$ (fourth outage) showed small growth in yaw error, reaching a maximum value of 0.5 degrees. During the fourth outage (470-530 s), 

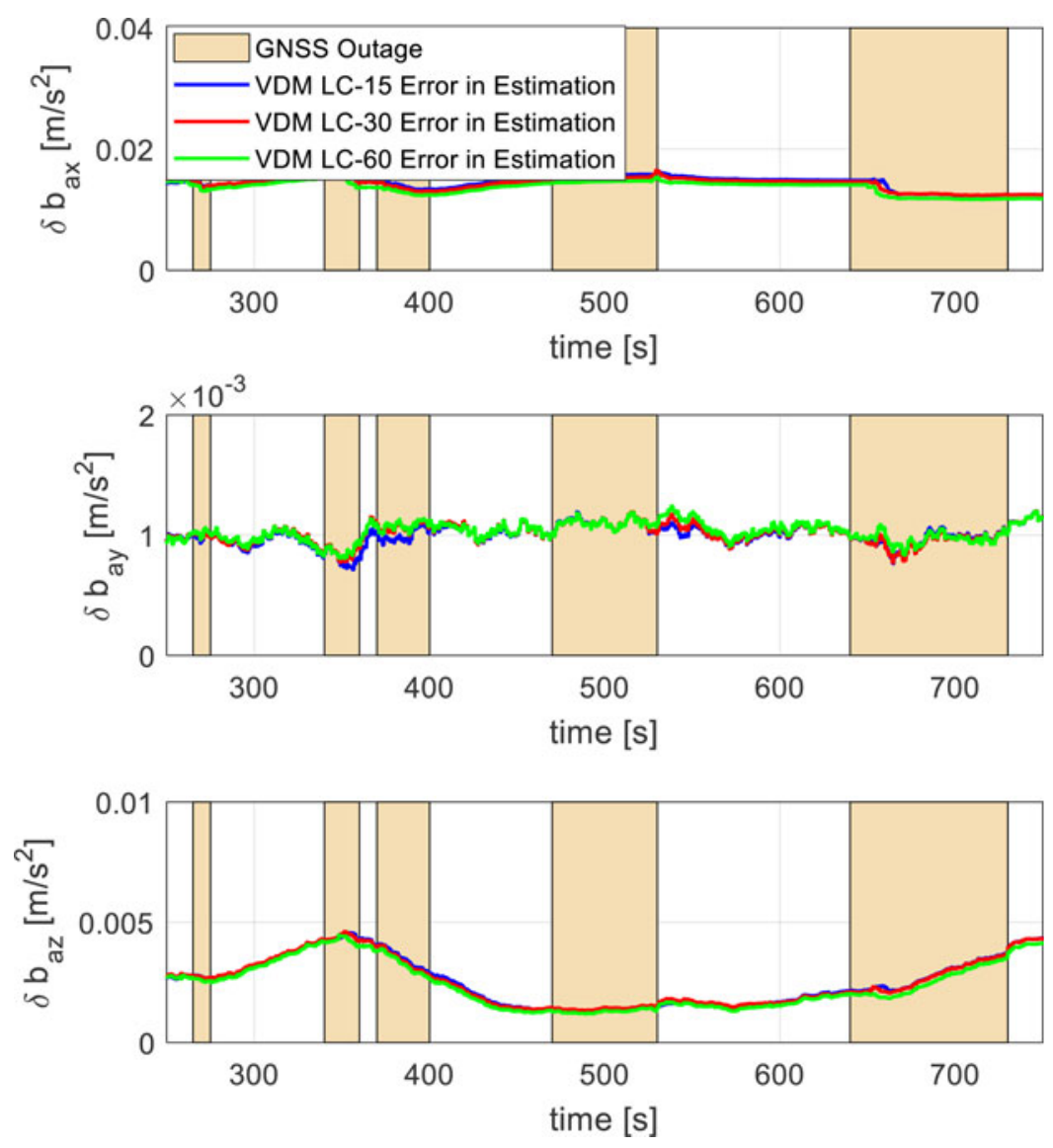

Figure 9. Accelerometer bias estimation errors for the VDM approach.

the aircraft experienced large rotation rates only during the last phase of the outage. Therefore, between $470 \mathrm{~s}$ and $520 \mathrm{~s}$, the aircraft was flying mostly straight and level, causing only slight growth in yaw angle error, as can be seen in Figure 8.

During a turn, the maximum rotation rate achieved was found to influence roll angle errors the most and pitch angle errors to a lesser extent. With a rate limit of $15 \%$, the roll angle error reached a maximum of 0.17 degrees $(654 \mathrm{~s})$, while for $30^{\circ}$ s s it reached a maximum of 0.36 degrees $(650 \mathrm{~s})$ and reached a maximum of 0.46 degrees $(648 \mathrm{~s})$ with a rate limit of $60^{\circ} / \mathrm{s}$. With the VDM approach, the roll angle error quickly recovered after short periods of rapid roll dynamics. The large increase in roll angle error during sections with large rotation rates occurred even with GNSS availability with the VDM approach, as shown in the interval between $250 \mathrm{~s}$ and $265 \mathrm{~s}$. The large instantaneous error, correlated with the rotation rate, is mainly attributed to the remaining part of the initialisation errors, especially in the VDM parameters. The use of the VDM prevented further growth of the attitude errors following rapid dynamics even in periods of extended GNSS outage lasting $90 \mathrm{~s}$. On the other hand, with an INS/GNSS approach, the attitude errors grew rapidly, with the maximum error observed being correlated with the length of the outage period.

The accelerometer bias estimation error is presented in Figure 9. After filter convergence, the accelerometer bias estimation errors remained bounded and did not grow during the GNSS outages. The simple random walk model used to estimate the accelerometer bias provided reasonable results enabling good navigation performance for a flight period lasting $780 \mathrm{~s}$ with short periods of GNSS outage in between. Large roll rotation rates during an outage period did not seem to influence the estimation performance of the accelerometer bias following the convergence of the filter. 

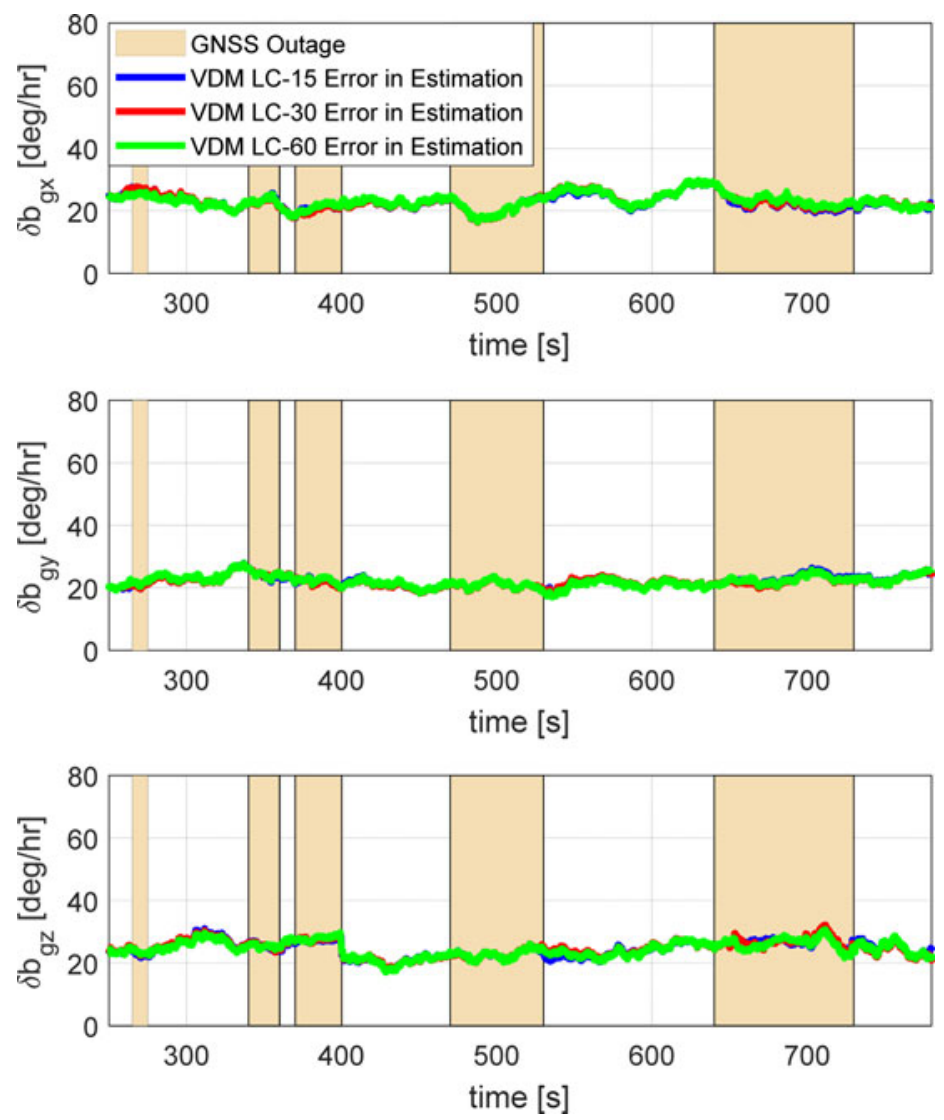

Figure 10. Gyroscope bias estimation errors for the VDM approach.

The gyroscope bias estimation error is presented in Figure 10. It was found that $98 \%$ of the initial turn-on gyroscope bias and bias variation was resolved well within $100 \mathrm{~s}$ of GNSS presence. And just like the accelerometer bias, GNSS outages of different lengths, $10 \mathrm{~s}, 20 \mathrm{~s}, 30 \mathrm{~s}, 60 \mathrm{~s}$ and $90 \mathrm{~s}$, did not seem to influence the estimation error of the gyroscope bias owing to the use of the VDM and direct IMU measurements during the outage. Further, large roll rotation rates reaching $60 \%$ s during the GNSS outages did not seem to influence the estimation of the gyroscope bias following the convergence of the filter.

The RMS of the mean error of 22 VDM parameters is presented in Figure 11. A GNSS outage alone lasting $10 \mathrm{~s}, 20 \mathrm{~s}, 30 \mathrm{~s}, 60 \mathrm{~s}$ or $90 \mathrm{~s}$ did not seem to influence the VDM parameter estimation error. However, turning during a GNSS outage actually led to improved observability of the VDM parameters, thanks to the availability of IMU measurements during this period. Further, a low roll rotation rate of $15 \%$ led to slightly better observability of VDM parameters as opposed to $60 \%$, but the difference was just $0.5 \%$.

Figure 12 shows the RMS of wind magnitude errors. The VDM approach provided the capability to estimate wind velocity, which in turn improved the navigation solution during GNSS outages as opposed to an INS/GNSS approach. It was found that GNSS outages lasting less than $60 \mathrm{~s}$ did not have a significant influence on the estimated wind magnitude error. With a GNSS outage lasting $90 \mathrm{~s}$ (fifth outage), the error in the estimated wind magnitude was found to grow gradually, reaching $0.2 \mathrm{~m} / \mathrm{s}$ at the end of the fifth outage. The level of dynamics, especially in the roll axis, seemed not to influence the growth of wind magnitude error significantly. 


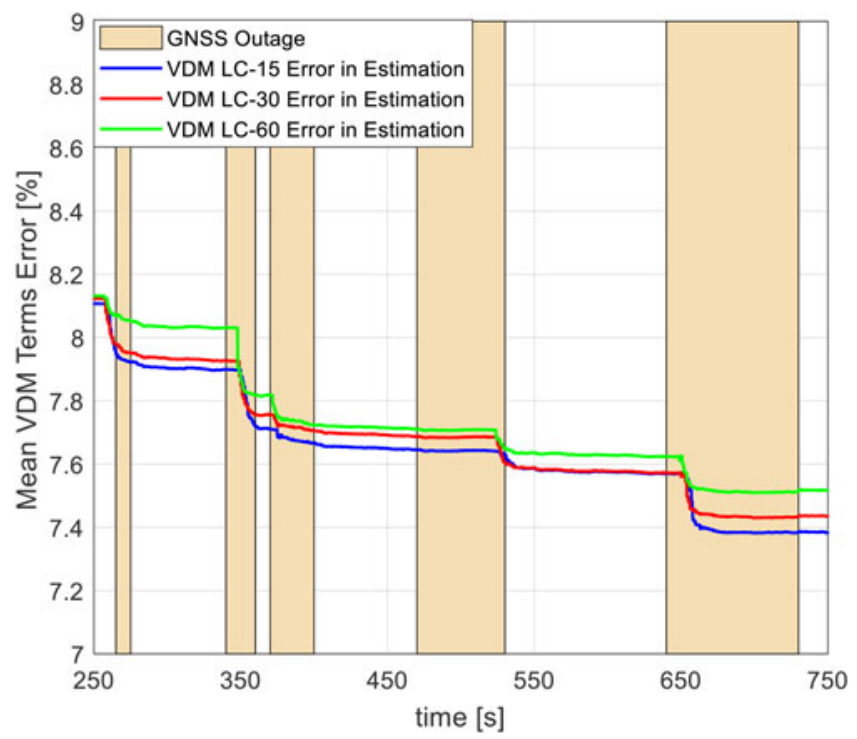

Figure 11. VDM parameters estimation error.

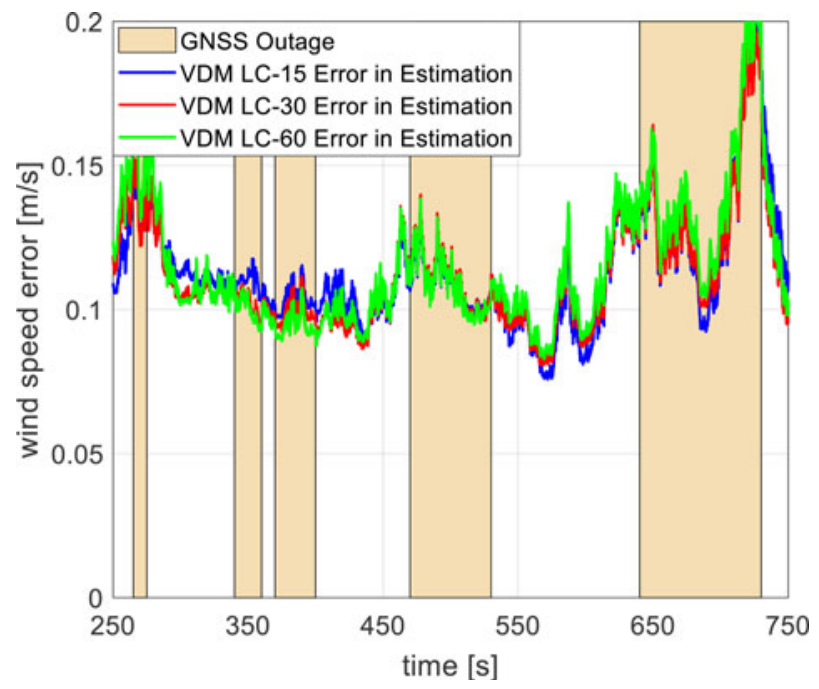

Figure 12. Wind speed error for VDM.

\section{Conclusions}

The error characteristics of a VDM approach using IMU and GNSS measurements have been presented. The position error was found to grow proportionally with roll rate for an extended GNSS outage lasting $90 \mathrm{~s}$. Attitude errors were not significantly influenced by GNSS outages lasting up to $60 \mathrm{~s}$, with extended outages $(90 \mathrm{~s})$ mainly influencing yaw error. Further, it was found that VDM parameters remain observable during a GNSS outage provided the aircraft manoeuvres during this period. Also, it was found that the level of dynamics in the roll axis did not seem to influence the growth of wind magnitude errors significantly. The presented architecture has shown superior navigation performance with varying roll rates as opposed to an INS/GNSS approach operating with a fairly modest rate of $15^{\circ} / \mathrm{s}$ during GNSS outages. The approach has the potential to work alongside and even replace (at certain times, such as during an extended GNSS outage) the conventional INS/GNSS integration, especially 
in low-cost applications where the aircraft could experience rapid dynamics or multipath path effects causing GNSS outages. Such applications include aerial mapping and surveying, inspection of wind turbines, and search and rescue operations in mountainous areas. The real-time implementation of the architecture is the subject of future research, including further investigation into the influence of measurement delay and control input noise on the error characteristics.

Acknowledgements. The authors would like to thank the INNOVATIVE doctoral programme for funding the work. The INNOVATIVE programme is partially funded by the Marie Curie Initial Training Networks (ITN) action (project number 665468) and partially by the Institute for Aerospace Technology (IAT) at the University of Nottingham.

\section{References}

Bryson, M. and Sukkarieh, S. (2004). Vehicle Model Aided Inertial Navigation for a UAV Using Low-Cost Sensors. Proceedings of the Australasian Conference on Robotics and Automation (ACRA 04), Canberra, Australia, December 2004.

Crocoll, P., Seibold, J., Scholz, G. and Trommer, G. F. (2014). Model-aided navigation for a quadrotor helicopter: A novel navigation system and first experimental results. Navigation, 61(4), 253-271.

Dadkhah, N., Mettler, B. and Gebre-egziabher, D. (2008). A Model-Aided AHRS for Micro Aerial Vehicle Application. Proceedings of the 21st International Technical Meeting of the Satellite Division of The Institute of Navigation (ION GNSS 2008), Savannah, GA, pp. 545-553.

Ducard, G. (2007). Fault-tolerant flight control and guidance systems for a small unmanned aerial vehicle. Doctor of Sciences dissertation, ETH Zürich, Switzerland. doi:10.3929/ethz-a-010782581

El-Diasty, M. and Pagiatakis, S. (2009). A rigorous temperature-dependent stochastic modelling and testing for MEMS-based inertial sensor errors. Sensors, 9(11), 8473-8489. doi:10.3390/s91108473

George, M. and Sukkarieh, S. (2005). Tightly Coupled INS/GPS with Bias Estimation for UAV Applications. Proceedings of the Australasian Conference on Robotics and Automation (ACRA 05), Sydney, Australia, December 2005.

Groves, P. D. (2008). Principles of GNSS, Inertial, and Multisensor Integrated Navigation Systems. Boston and London: Artech House. doi:10.1109/MAES.2014.14110

Hide, C. (2003). Integration of GPS and low cost INS measurements. PhD thesis. University of Nottingham, UK.

Jospin, L., Stoven-Dubois, A. and Cucci, D. A. (2019). Photometric long-range positioning of LED targets for cooperative navigation in UAVs. Drones, 3(3), 69. doi:10.3390/drones3030069

Khaghani, M. and Skaloud, J. (2016). Autonomous vehicle dynamic model-based navigation for small UAVs. Navigation, 63(3), 345-358. doi:10.1002/navi.140

Khaghani, M. and Skaloud, J. (2018). VDM-based UAV Attitude Determination in Absence of IMU Data. European Navigation Conference. ENC, 2018. IEEE, pp. 84-90. doi:10.1109/EURONAV.2018.8433243.

Kim, J. and Sukkarieh, S. (2003). A Baro-Altimeter Augmented INS/GPS Navigation System for an Uninhabited Aerial Vehicle. The 6th International Symposium on Satellite Navigation Technology Including Mobile Positioning \& Location Services (SATNAV 03), Melbourne, Australia, July 2003.

Koifman, M. and Bar-Itzhack, I. Y. (1999). Inertial navigation system aided by aircraft dynamics. IEEE Transactions on Control Systems Technology, 7(4), 487-493. doi:10.1109/87.772164

Lau, T. K., Liu, Y. H. and Lin, K. W. (2013). Inertial-based localization for unmanned helicopters against GNSS outage. IEEE Transactions on Aerospace and Electronic Systems, 49(3), 1932-1949. doi:10.1109/TAES.2013.6558029

Mueller, K., Crocoll, P. and Trommer, G. F. (2016). Model-Aided Navigation with Wind Estimation for Robust Quadrotor Navigation. Proceedings of the 2016 International Technical Meeting of The Institute of Navigation, Monterey. California, pp. 689-696. doi:10.33012/2016.13466

Mwenegoha, H., Moore, T., Pinchin, J. and Jabbal, M. (2019). Model-based autonomous navigation with moment of inertia estimation for unmanned aerial vehicles. Sensors, 19, 11. doi: 10.3390/s19112467

Papadimitratos, P. and Jovanovic, A. (2008). Protection and Fundamental Vulnerability of GNSS. 2008 International Workshop on Satellite and Space Communications, IWSSC'08, Conference Proceedings, pp. 167-171. doi:10.1109/IWSSC.2008.4656777.

Quinchia, A. G., Falco, G., Falletti, E., Dovis, F. and Ferrer, C. (2013). A comparison between different error modeling of MEMS applied to GPS/INS integrated systems. Sensors (Switzerland), 13(8), 9549-9588. doi:10.3390/s130809549

Robustelli, U. and Pugliano, G. (2018). GNSS code multipath short-time Fourier transform analysis. Navigation, 65(3), 353-362. doi:10.1002/navi.247

Sendobry, A. (2014). Control system theoretic approach to model based navigation. Doctoral thesis. Technische Universität Darmstadt, doi:10.13140/RG.2.2.22171.87846

Tawk, Y., Tomé, P., Botteron, C., Stebler, Y. and Farine, P.-A. (2014). Implementation and performance of a GPS/INS tightly coupled assisted PLL architecture using MEMS inertial sensors. Sensors, 14(2), 3768-3796. doi: 10.3390/s140203768

Cite this article: Mwenegoha HA, Moore T, Pinchin J, Jabbal M (2021). Error characteristics of a model-based integration approach for fixed-wing unmanned aerial vehicles. The Journal of Navigation 74: 6, 1353-1366. https://doi.org/10.1017/S0373463321000424 